\title{
Languages recognized with unbounded error by quantum finite automata
}

\author{
Abuzer Yakaryllmaz and A.C. Cem Say \\ Bog̃aziçi University, Department of Computer Engineering, \\ Bebek 34342 İstanbul, Turkey \\ abuzer, say@boun.edu.tr *
}

February 22, 2019

\begin{abstract}
We prove the following facts about the language recognition power of KondacsWatrous quantum finite automata in the unbounded error setting: One-way automata of this kind recognize all and only the stochastic languages. When the tape head is allowed two-way (or even "1.5-way") movement, more languages become recognizable. This leads to the conclusion that quantum Turing machines are more powerful than probabilistic Turing machines when restricted to constant space bounds.
\end{abstract}

\section{Introduction}

Several alternative models $[1,2,4,10,13,18,20]$ of quantum finite automata (QFA's) have been studied in the recent years. Most of the attention in this regard has been focused on the classes of languages recognized by these machines with bounded error $[1,3,8,10,13,15,16]$. In this paper, we examine the computational power of one of the most popular QFA models, the measure-many (Kondacs-Watrous) QFA, in the unbounded error setting. We give a complete characterization of the class of languages recognized by one-way QFA's of this kind; they turn out to recognize all and only the stochastic languages. We also show that allowing the tape head to "stay put" for some steps during its left-toright traversal of the input increases the language recognition power of these QFA's. This contrasts the situation in the classical probabilistic models, where two-way and one-way automata are equivalent in power in this setting [12]. We conclude that quantum Turing machines are strictly more powerful than their probabilistic counterparts when restricted to constant space bounds.

The rest of this paper is structured as follows: Section 2 contains the relevant background information. Section 3 presents our results. Section 4 is a conclusion.

\section{Preliminaries}

In this section, we give a brief review of the definitions and facts that will be used in the rest of the paper.

\subsection{Classical Automata}

A 1-way probabilistic finite automaton (1pfa) [23] with $n \in \mathbb{Z}^{+}$states is a 5 tuple $\mathcal{P}=\left(S, \Sigma,\left\{\mathrm{A}_{\sigma} \mid \sigma \in\right.\right.$ $\left.\Sigma\}, \mathrm{v}_{0}, F\right)$, where

1. $S=\left\{s_{1}, \cdots, s_{n}\right\}$ is the set of states,

2. $\Sigma$ is the finite input alphabet,

3. $\mathrm{A}_{\sigma}$ is the $n \times n$ real-valued stochastic transition matrix for symbol $\sigma$, that is, $\mathrm{A}_{\sigma}(i, j)$ is the value of the transition probability from state $s_{i}$ to state $s_{j}$ when reading symbol $\sigma$,

\footnotetext{
^ This work was partially supported by the Bog̃aziçi University Research Fund with grant 08A102.
} 
4. $\mathrm{v}_{0}$ is a $1 \times n$ vector representing the initial distribution of the states, that is, the $i^{\text {th }}$ entry of $\mathrm{v}_{0}$ is the probability that $\mathcal{P}$ is initially in state $s_{i}$, and,

5. $F \subseteq S$ is the set of accepting states.

For an input string $w \in \Sigma^{*}$, the computation of $\mathcal{P}$ can be traced using the relations

$$
\begin{array}{r}
\mathrm{v}_{j}=\mathrm{v}_{j-1} \mathrm{~A}_{w_{j}} \\
\mathrm{~A}_{w}=\mathrm{A}_{w_{1}} \mathrm{~A}_{w_{2}} \cdots \mathrm{A}_{w_{|w|}} \\
\mathrm{v}_{|w|}=\mathrm{v}_{0} \mathrm{~A}_{w}
\end{array}
$$

where $w_{i}$ is the $i^{t h}$ symbol of $w$ and $v_{j}$ is the vector of states after step $j(1 \leq i, j \leq|w|)$. The acceptance probability of $w$ is

$$
f_{\mathcal{P}}(w)=\sum_{s_{i} \in F} \mathrm{v}_{|w|}(i),
$$

where $\mathrm{v}_{|w|}(i)$ denotes the $i^{t h}$ entry of $\mathrm{v}_{|w|}$. The language $L \subseteq \Sigma^{*}$ recognized by $\mathcal{P}$ with cut-point $\lambda \in[0,1)$ is defined as

$$
L=\left\{w \mid w \in \Sigma^{*}, f_{\mathcal{P}}(w)>\lambda\right\} .
$$

Languages recognized by 1PFA's with cut-point form the class of stochastic languages.

Theorem 1. [22] If $L$ is a stochastic language, then for any $\lambda \in(0,1)$, there exists a 1PFA that recognizes $L$ with cut-point $\lambda$.

The generalized probabilistic finite automaton (GPFA) [24] model is a generalization of the 1PFA model where neither the transition matrices nor the state vectors need to be stochastic. Additionally, a GPFA has a final vector $\mathrm{f}$, which is a column vector with real entries, instead of the set of accepting states. Formally, a generalized probabilistic finite automaton (GPFA) with $n \in \mathbb{Z}^{+}$states is a 5 tuple $\mathcal{G}=\left(S, \Sigma,\left\{\mathrm{A}_{\sigma} \mid \sigma \in \Sigma\right\}, \mathrm{v}_{0}, \mathrm{f}\right)$, where

1. $S$ is the set of $n$ states,

2. $\Sigma$ is the finite input alphabet,

3. $\mathrm{A}_{\sigma}$ is the $n \times n$ real-valued transition matrix for symbol $\sigma$,

4. $\mathrm{v}_{0}$ is the real-valued initial $1 \times n$ vector,

5 . and $\mathrm{f}$ is the real-valued final $n \times 1$ vector.

For an input string $w \in \Sigma^{*}$, the acceptance probability of $w$ by GPFA $\mathcal{G}$ is calculated as

$$
f_{\mathcal{G}}(w)=\mathrm{v}_{0} \mathrm{~A}_{w} \mathrm{f}=\mathrm{v}_{|w|} \mathrm{f}
$$

where $\mathrm{A}_{w}$ is as defined in Equation 2. Note that the range of $f_{\mathcal{G}}$ is $\mathbb{R}$. The language $L \subseteq \Sigma^{*}$ recognized by $\mathcal{G}$ with cut-point $\lambda \in \mathbb{R}$ is defined as

$$
L=\left\{w \mid w \in \Sigma^{*}, f_{\mathcal{P}}(w)>\lambda\right\}
$$

It is known that the class of languages recognized by GPFA's with cut-point is precisely the class of stochastic languages [24].

Theorem 2. [24] If $L$ is recognized with cut-point $\lambda_{1} \in \mathbb{R}$ by a GPFA with $n$ states, then there exist a 1PFA $\mathcal{P}$ with $O\left(n^{2}\right)$ states and a cut-point $\lambda_{2} \in[0,1)$ such that $\mathcal{P}$ recognizes $L$ with cut-point $\lambda_{2}$.

Another generalization of the 1PFA is the two-way probabilistic automaton (2PFA) model [14], in which the input string is viewed as written on a tape flanked by two end-markers, and the machine has a tape head which can move to the left or stay put, as well as moving to the right. This additional capability does not increase the recognition power; the class of languages recognized by 2PFA's with cut-point is again equal to the stochastic languages [12]. 


\subsection{Quantum Automata}

A 1-way Kondacs-Watrous quantum finite automaton (KWQFA) [13] with $n \in \mathbb{Z}^{+}$states is a 5-tuple $\mathcal{M}=\left(Q, \Sigma,\left\{\mathrm{U}_{\sigma} \mid \sigma \in \Sigma \cup\{\Phi, \$\}\right\}, Q_{a c c}, Q_{r e j}\right)$, where

1. $Q=\left\{q_{1}, \cdots q_{n}\right\}$ is the set of states, such that $q_{1}$ is the initial state,

2. $\Sigma$ is the finite input alphabet not containing the symbols $\$$ and $\$$,

3. $\mathrm{U}_{\sigma}$ is the $n \times n$ complex-valued unitary transition matrix for symbol $\sigma \in \Sigma \cup\{\phi, \$\}$ such that $\mathrm{U}_{\sigma}(j, i)=$ $\alpha$ if the amplitude of the transition from $q_{i}$ to $q_{j}$ is $\alpha$ when reading the symbol $\sigma$,

4. $Q_{a c c}$ and $Q_{r e j}$, disjoint subsets of $Q$, are the sets of accepting and rejecting states.

Additionally, $Q_{n o n}=Q \backslash\left(Q_{a c c} \cup Q_{r e j}\right)$ is the set of non-halting states; $\mathrm{P}_{\text {non }}, \mathrm{P}_{\text {acc }}$, and $\mathrm{P}_{\text {rej }}$ are diagonal zero-one projection matrices, which project the state vector onto the subspaces of non-halting, accepting, and rejecting states, such that $\mathrm{P}_{\text {non }}(j, j)=1$ if $q_{j} \in Q_{n o n}, \mathrm{P}_{\text {acc }}(j, j)=1$ if $q_{j} \in Q_{a c c}$, and $\mathrm{P}_{\text {rej }}(j, j)=1$ if $q_{j} \in Q_{r e j}$, respectively; $\Gamma=\Sigma \cup\{\phi, \$\}$ is the tape alphabet where $\mathbb{\phi}$ and $\$$ are the end-markers, and for input string $w \in \Sigma^{*}$, the tape contains $₫ w \$$.

The state vector, by an unfortunate twist of convention between probabilistic and quantum automata, is a column vector, and is denoted as $|\mathbf{u}\rangle$. Its conjugate transpose is a row vector, and is denoted by $\langle\mathbf{u}|$.

The computation of $\mathcal{M}$ proceeds as follows: $\left|\mathbf{u}_{0}\right\rangle$ is the initial state vector, where $\left|\mathbf{u}_{0}\right\rangle(1)=1$ and all other entries are zeros, and $\left|\mathbf{u}_{0}^{n}\right\rangle=\left|\mathrm{u}_{0}\right\rangle$. For a given input string $w \in \Sigma^{*}, \mathbf{w}=\boldsymbol{c} w \$$,

$$
\begin{array}{r}
\left|\mathbf{u}_{i}^{n}\right\rangle=P_{\text {non }}\left|\mathbf{u}_{i}\right\rangle,\left|\mathbf{u}_{i}^{a}\right\rangle=P_{\text {acc }}\left|\mathbf{u}_{i}\right\rangle,\left|\mathbf{u}_{i}^{r}\right\rangle=\mathrm{P}_{\text {rej }}\left|\mathbf{u}_{i}\right\rangle, \\
\left|\mathbf{u}_{i}\right\rangle=\mathrm{U}_{\mathbf{w}_{i}\left|\mathbf{u}_{i-1}^{n}\right\rangle}, \\
P_{\mathcal{M}, a c c}(i)=P_{\mathcal{M}, a c c}(i-1)+\left\langle\mathbf{u}_{i}^{a} \mid \mathbf{u}_{i}^{a}\right\rangle \\
P_{\mathcal{M}, r e j}(i)=P_{\mathcal{M}, r e j}(i-1)+\left\langle\mathbf{u}_{i}^{r} \mid \mathbf{u}_{i}^{r}\right\rangle
\end{array}
$$

where $1 \leq i \leq|\mathbf{w}| ;\left|\mathbf{u}_{i}\right\rangle$ is the state vector after the $i^{t h}$ step; $P_{\mathcal{M}, a c c}$ and $P_{\mathcal{M}, \text { rej }}$ are finite sequences that trace the acceptance and rejection probabilities that have accumulated so far during the computation, with initial values $P_{\mathcal{M}, a c c}(0)=0$ and $P_{\mathcal{M}, r e j}(0)=0$, respectively. Equations 4 and 5 mean that the machine undergoes two operations in each step. First, its state vector evolves according to the unitary transformation dictated by the scanned symbol. Then, it is measured to see whether it has accepted, rejected, or not halted yet. As seen in Equations 6 and 7, the acceptance and rejection probabilities are calculated using the amplitudes of the relevant states. Halting states "drop out" of the state vector, and computation continues with only the non-halting states having nonzero amplitude.

The probability that $\mathcal{M}$ accepts input $w$ is

$$
f_{\mathcal{M}}(w)=P_{\mathcal{M}, a c c}(|\phi w \$|),
$$

and the language $L \subseteq \Sigma^{*}$ recognized by $\mathcal{M}$ with cut-point $\lambda \in[0,1)$ is defined as

$$
L=\left\{w \mid w \in \Sigma^{*}, f_{\mathcal{M}}(w)>\lambda\right\} .
$$

The class of languages recognized by KWQFA's with cut-point (i.e. with unbounded error) has been studied by Brodsky and Pippenger [10], who named it UMM, and proved some closure properties ${ }^{1}$. Analogously to Theorem 1, any language in UMM can be recognized by a KWQFA with cutpoint $\frac{1}{2}$.

KWQFA's can be generalized by allowing the tape head more freedom of movement.

A 1.5-way quantum finite automaton (1.5QFA) [1] can be seen as a generalized KWQFA where the tape head is allowed to stay put, and does not have to move right in all steps. The transition function $\delta: Q \times \Gamma \times Q \times\{0,1\} \rightarrow \mathbb{C}$ of a 1.5QFA, where $Q$ and $\Gamma$ are as defined above, is interpreted as follows: For each $q, q^{\prime} \in Q, \delta \in \Gamma$ and $d \in\{0,1\}, \delta\left(q, \sigma, q^{\prime}, d\right)$ is the amplitude with which the machine

\footnotetext{
${ }^{1}$ Note that the proof in [10] that UMM is closed under complementation contains an error.
} 
currently in state $q$ and scanning symbol $\sigma$ will change to state $q^{\prime}$ and move its head $d$ symbols to the right.

Not every transition function of the form described above can be used in a 1.5QFA. The additional restrictions imposed by quantum theory on $\delta$ are described in detail in [13]. In the particular 1.5QFA we will describe in Section 3, every transition entering the same state involves the tape head moving in the same direction (either right or stationary). We represent this feature of a state $q$ using the appropriate one of the notations $\vec{q}, \downarrow q$ for this state in the machine description. With this simplification, considering the Hilbert space $\ell_{2}(Q)$, a syntactically correct $1.5 \mathrm{QFA}$ can be specified easily by just providing a unitary operator $U_{\sigma}: \ell_{2}(Q) \rightarrow \ell_{2}(Q)$ for each symbol $\sigma \in \Gamma$, exactly as described earlier for one-way KWQFA's. $\delta$ can then be specified to be just

$$
\delta\left(q, \sigma, q^{\prime}, d\right)=\left\{\begin{array}{cc}
\left\langle q^{\prime}\left|U_{\sigma}\right| q\right\rangle & \text { if } D\left(q^{\prime}\right)=d \\
0 & \text { if } D\left(q^{\prime}\right) \neq d
\end{array},\right.
$$

where the function $D: Q \rightarrow\{0,1\}$ maps each state to the single direction in which all incoming transitions to this state move the tape head.

A pair of the form (state, head position) is called a configuration of a 1.5QFA. A 1.5QFA which is working on a tape of $n$ symbols (including the end-markers) has therefore $n|Q|$ different configurations. Initially, the head is on the left end-marker, and so the machine starts in the configuration $\left(q_{0}, 0\right)$. At later steps of the computation, the machine may exist in superpositions of more than one configuration. It is sometimes useful to visualize such superpositions of multiple configurations as snapshots of the machine running in multiple parallel computation paths.

As described above for KWQFA's, each step of the computation consists of a unitary transformation of the current superposition according to the transition function, followed by a measurement to see whether the machine has accepted, rejected, or not halted yet. The probability of each outcome is determined by the amplitudes of the relevant configurations in the present superposition. The observation of accepting (rejecting) configurations cause the accumulated acceptance (rejection) probability to be updated accordingly. The machine continues running from a superposition of the non-halting configurations.

In the 2-way quantum finite automaton (2QFA) [13] model, the tape head is allowed to go left, as well as staying put and going to the right, and the exposition above regarding 1.5QFA's can be generalized in a straightforward manner to 2QFA's.

\section{Main Results}

Our first result is a complete characterization of UMM.

Lemma 1. Any language recognized with cutpoint $\frac{1}{2}$ by a 1 PFA with $n$ states can be recognized with cutpoint $\frac{1}{2}$ by a KWQFA with $O\left(n^{2}\right)$ states.

Proof. Let $\mathcal{P}=\left(S, \Sigma,\left\{\mathrm{A}_{\sigma} \mid \sigma \in \Sigma\right\}, \mathbf{v}_{0}, F\right)$ be a 1 PFA with $n$ states that recognizes language $L$ with cutpoint $\frac{1}{2}$. We construct a KWQFA $\mathcal{M}=\left(Q, \Sigma,\left\{\mathrm{U}_{\sigma} \mid \sigma \in \Sigma \cup\{\Phi, \$\}\right\}, Q_{a c c}, Q_{\text {rej }}\right)$ with $O\left(n^{2}\right)$ states that recognizes the same language with cutpoint $\frac{1}{2}$.

For each $s_{i} \in S$, there will be a corresponding non-halting state $q_{i} \in Q_{\text {non }}(1 \leq i \leq n)$. $\mathcal{M}$ will have two special halting states, $q_{n+1} \in Q_{a c c}$ and $q_{n+2} \in Q_{r e j}$. The remaining states of $\mathcal{M}$ are accepting and rejecting states that will be defined later.

The idea behind the construction is to convert the stochastic transition matrices of $\mathcal{P}$ to unitary transition matrices of $\mathcal{M}$ by adding new rows and columns (corresponding to new halting states), so that the distribution of the probabilities in the state vector of $\mathcal{P}$ is "imitated" by the distribution of the amplitudes of the non-halting states of $\mathcal{M}$ : For any position $t$ of the input string, there will be a positive real number $k_{t}$ such that the amplitude of $q_{i}$ just after $\mathcal{M}$ reads the $t^{t h}$ symbol equals $k_{t}$ times the probability of $s_{i}$ just after $\mathcal{P}$ reads that symbol $(1 \leq i \leq n)$. The newly added halting states 
will come in accept/reject pairs, so that transitions to them during the computation will add equal amounts to the overall acceptance and rejection probabilities, and therefore will not affect the decision on the membership of the input in $L$.

We begin the construction by defining two stochastic matrices $A_{\mathscr{C}}$ and $A_{\Phi}$ for the symbols $\Phi$ and $\$$, respectively. We define $\mathrm{A}_{\mathbb{C}}$ as if $\mathcal{P}$ started in state $s_{1}$, and then made a transition to its initial state vector $v_{0}$ when reading symbol $\phi$. For this purpose, it is enough to set the first row of $A_{\mathbb{C}}$ as $v_{0}$. (The remaining rows can be arbitrary stochastic vectors.) We would like the acceptance and rejection probabilities of $\mathcal{P}$ at the end of the computation to accumulate in a single accept and a single reject state, respectively. Therefore, $\mathrm{A}_{\$}$ is defined as if $\mathcal{P}$ had two more states, $s_{n+1} \in F$ and $s_{n+2} \in S \backslash F$, and the probability of the transitions from all accept states to $s_{n+1}$ and those from all other states to $s_{n+2}$ were 1 when reading symbol $\$$. (Note that, $q_{n+1}$ and $q_{n+2}$ in $\mathcal{M}$ will correspond to $s_{n+1}$ and $s_{n+2}$, respectively.) The matrices $\mathrm{A}_{\sigma}$ for $\sigma \in \Sigma \cup\{\boldsymbol{\phi}, \$\}$ are extended so that both $s_{n+1}$ and $s_{n+2}$ have self-loops with transition probability 1.

Now, for each symbol $\sigma \in \Sigma \cup\{\Phi, \$\}$, we construct a unitary matrix $\mathrm{U}_{\sigma}$, by starting with the stochastic one $\mathrm{A}_{\sigma}$, adding new states to it, and then updating all transition amplitudes carefully so that both unitarity is ensured, and $\mathcal{M}$ imitates $\mathcal{P}$ in the sense described above. This transformation consists of the following five stages:

1. Orthogonalization. Each pair of rows, say, $(i, j)$, corresponding to $\left(s_{i}, s_{j}\right),(i<j)$ belonging to $S \cup\left\{s_{n+1}, s_{n+2}\right\}$, are made orthogonal with each other. This is achieved by adding two new states, one in $Q_{a c c}$ and one in $Q_{r e j}$, to $\mathcal{M}$, and extending the matrices with the corresponding additional pairs of columns and rows. If the inner product $\left\langle\mathrm{A}_{\sigma}(i) \mid \mathrm{A}_{\sigma}(j)\right\rangle$ is $\alpha$, we define the entries at which rows $i$ and $j$ cross the two new columns of the new states as follows: Row $i$ contains $-\sqrt{\frac{\alpha}{2}}$ in both positions, whereas row $j$ contains $\sqrt{\frac{\alpha}{2}}$ in both positions. Therefore, the inner product of the two rows for $q_{i}$ and $q_{j}$ becomes 0 . All other entries in the top $n+2$ positions of the two newly added columns are 0's in order not to affect the inner products of other pairs of rows.

2. Padding. The lengths (norms) of all the $n+2$ rows orthogonalized in Stage 1 are equalized. At least one row has a maximum length $l$, and all shorter rows will be extended to this length. For each row with length $k<l$, two new states, (one in $Q_{a c c}$ and one in $Q_{r e j}$, as described above) are added to $\mathcal{M}$. The entries at which the row under consideration crosses these new columns are both set to $\sqrt{\frac{l^{2}-k^{2}}{2}}$, so that the length of the newly extended row equals $l$. Therefore, the lengths of all rows are equalized without changing the amplitudes inherited from the transition matrix of $\mathcal{P}$. As in Stage 1, all other entries in the top $n+2$ positions of the columns corresponding to the newly added states are 0 , so the orthogonality achieved in that stage is preserved.

3. Completion. The rows corresponding to the halting states added in Stages 1 and 2 are filled in, so that all rows of the matrix form an orthogonal set, and the length of each row is the same.

4. Normalization. All rows are normalized by dividing each entry with the square root of the common length, that is, all entries in the matrix associated with $\sigma$, including, of course, all those inherited from $\mathrm{A}_{\sigma}$, are multiplied by a constant $c_{\sigma}=\frac{1}{\sqrt{l}}$. At the end of this stage, the matrix is unitary.

5. Transposition. $U_{\sigma}$ is set to be the transpose of the matrix obtained above to accommodate for the switch between the matrix representations for probabilistic and quantum automata.

This construction requires at most $O\left(n^{2}\right)$ new states, and so $|Q|=O\left(n^{2}\right)$.

Note that, for each $\sigma \in \Sigma \cup\{\Phi, \$\}$, the matrix $\mathrm{U}_{\sigma}$ has the form

$$
\mathrm{U}_{\sigma}=\left(\frac{c_{\sigma} \mathrm{A}_{\sigma}^{\top}}{\mathrm{H}_{\sigma}} \mid \mathrm{B}_{\sigma}\right)
$$

where $\mathrm{A}_{\sigma}$ is the original stochastic matrix extended with $s_{n+1}$ and $s_{n+2}$ as previously described. $\mathrm{H}_{\sigma}$ and $\mathrm{B}_{\sigma}$ are created in Stages 1-2 and Stage 3, respectively, and then both are normalized and transposed to their present positions in Stages 4 and 5.

If $m$ pairs of halting states have been created during the transformation procedure, $\mathrm{H}_{\sigma}$ has $2 m$ rows. By our construction, for $1 \leq k \leq m$, the $(2 k-1)^{t h}$ and $(2 k)^{t h}$ rows of $\mathrm{H}_{\sigma}$ are identical vectors, 
corresponding to the incoming transition amplitudes of an accepting and a rejecting state of $\mathcal{M}$, respectively. Assuming that the $j^{\text {th }}$ symbol read during the computation is $\sigma$, the amplitudes of these $2 m$ states after that step can be determined by multiplying the last $2 m$ rows of $\mathrm{U}_{\sigma}$ with the non-halting states vector $\left|\mathbf{u}_{j-1}^{n}\right\rangle$ of the $(j-1)^{t h}$ step. Since only the top $n$ entries of $\left|\mathbf{u}_{j-1}^{n}\right\rangle$ can be nonzero, the contents of $\mathrm{B}_{\sigma}$ are immaterial to the calculation, and so $q_{2 k-1}$ and $q_{2 k}$ will always have exactly the same amplitude, and contribute equally to the overall acceptance and rejection probabilities.

For an input string $w \in \Sigma^{*}$, let $\mathbf{w}=\$ w \$$ denote the corresponding tape content including the endmarkers, and let $f_{\mathcal{P}}(w)$ and $f_{\mathcal{M}}(w)$ represent the acceptance probabilities of $\mathcal{P}$ and $\mathcal{M}$, respectively, for $w$. Recalling our notation from Section $2, \mathrm{v}_{i}$ is the $(n+2)$-dimensional state (row) vector of $\mathcal{P}$ after the $i^{t h}$ step of the computation, where $1 \leq i \leq|w| .\left|\mathbf{u}_{j}^{n}\right\rangle$ is the non-halting states vector of $\mathcal{M}$ after the $j^{\text {th }}$ step of its computation, where $1 \leq j \leq|\mathbf{w}|$. Before beginning to read $w, \mathcal{M}$ reads symbol c and we have

$$
\left|\mathbf{u}_{1}^{n}\right\rangle=c_{\mathbb{C}}\left(\mathbf{v}_{0} \mid 0, \cdots, 0\right)^{\top},
$$

where $\mathrm{v}_{0}$ is the initial state vector of $\mathcal{P}$. It is easily followed from $1,4,5,8$, and 9 that for any $i$,

$$
\left|\mathrm{u}_{i+1}^{n}\right\rangle=c_{\mathbb{C}}\left(\prod_{j=1}^{i} c_{w_{j}}\right)\left(\mathrm{v}_{i} \mid 0, \cdots, 0\right)^{\top}
$$

where $1 \leq i \leq|w|$, and $w_{j}$ denotes the $j^{t h}$ symbol of $w$. After reading symbol $\$$, the amplitude of $q_{n+1}$ is $\mathbf{c} f_{\mathcal{P}}(w)$, where $\mathbf{c}=\prod_{i=1}^{|\mathbf{w}|} c_{\mathbf{w}_{i}}$, recalling that the overall acceptance probability of $\mathbf{v}_{|w|}$, i.e., $f_{\mathcal{P}}(w)$, is summed up in $s_{n+1}$ by the transitions in $\mathrm{A}_{\$}$. Similarly, the amplitude of $q_{n+2}$ at that point equals $\mathbf{c}\left(1-f_{\mathcal{P}}(w)\right)$. As discussed above, at each step of the computation, some of the amplitude may be diverted to accepting and rejecting states, (except $q_{n+1}$ and $q_{n+2}$, which are reachable only when $\$$ is scanned), such that the newly registered probabilities of acceptance and rejection are equal. The total probability that the computation will halt in this manner in a state other than $q_{n+1}$ and $q_{n+2}$ is $1-\mathbf{c}^{2}\left(f_{\mathcal{P}}(w)\right)^{2}-\mathbf{c}^{2}\left(1-f_{\mathcal{P}}(w)\right)^{2}$. Therefore, the total acceptance probability of $\mathcal{M}$ is

$$
\begin{gathered}
f_{\mathcal{M}}(w)=\frac{1-\mathbf{c}^{2}\left(f_{\mathcal{P}}(w)\right)^{2}-\mathbf{c}^{2}\left(1-f_{\mathcal{P}}(w)\right)^{2}}{2}+\mathbf{c}^{2}\left(f_{\mathcal{P}}(w)\right)^{2}, \\
f_{\mathcal{M}}(w)=\frac{1}{2}+\frac{\mathbf{c}^{2}}{2}\left(2 f_{\mathcal{P}}(w)-1\right),
\end{gathered}
$$

where $0<\mathbf{c} \leq 1$. If $w \in L$, then $f_{\mathcal{P}}(w)>\frac{1}{2}$, and so $f_{\mathcal{M}}(w)>\frac{1}{2}$. If $w \notin L$, then $f_{\mathcal{P}}(w) \leq \frac{1}{2}$, and so $f_{\mathcal{M}}(w) \leq \frac{1}{2}$.

The standard definitions of PFA's and QFA's that we gave in Section 2 allow arbitrary real numbers as transition amplitudes, and therefore the related language classes include undecidable languages. To rectify this situation, one may restrict the amplitudes to efficiently computable numbers, as in [6]. Our results in this section remain valid in that case as well.

Lemma 2. Let $\mathcal{M}$ be a KWQFA with $n$ states and $f_{\mathcal{M}}: \Sigma^{*} \rightarrow[0,1]$ be its acceptance probability function. Then there exists a GPFA $\mathcal{G}$ with $O\left(n^{2}\right)$ states such that $f_{\mathcal{M}}(w)=f_{\mathcal{G}}(w)$ for all $w \in \Sigma^{*}$.

Proof. Let $\mathcal{M}=\left(Q, \Sigma,\left\{\mathrm{U}_{\sigma} \mid \sigma \in \Sigma \cup\{\Phi, \$\}\right\}, Q_{a c c}, Q_{r e j}\right)$ and $\left|Q_{\text {non }}\right|=k_{1},\left|Q_{a c c}\right|=k_{2},\left|Q_{r e j}\right|=k_{3}$, where $k_{1}, k_{2}, k_{3} \geq 0$ and $k_{1}+k_{2}+k_{3}=n$. We assume that the states in $Q$ are indexed as

$-q_{i} \in Q_{\text {non }}$ for $1 \leq i \leq k_{1}$,

$-q_{i} \in Q_{a c c}$ for $k_{1}<i \leq k_{1}+k_{2}$,

$-q_{i} \in Q_{r e j}$ for $k_{1}+k_{2}<i \leq n$.

For any symbol $\sigma \in \Sigma \cup\{\Phi, \$\}\}, \mathrm{U}_{\sigma}$ can be partitioned into nine blocks as

$$
\mathrm{U}_{\sigma}=\left(\begin{array}{c|c|c}
\mathrm{U}_{\sigma, n-n} & \mathrm{U}_{\sigma, a-n} & \mathrm{U}_{\sigma, r-n} \\
\hline \mathrm{U}_{\sigma, n-a} & \mathrm{U}_{\sigma, a-a} & \mathrm{U}_{\sigma, r-a} \\
\hline \mathbf{U}_{\sigma, n-r} & \mathbf{U}_{\sigma, a-r} & \mathbf{U}_{\sigma, r-r}
\end{array}\right),
$$


where $U_{\sigma, \mathfrak{a}-\mathfrak{b}}$ represents the transitions from the states in $Q_{\mathfrak{a}}$ to the states in $Q_{\mathfrak{b}}, \mathfrak{a}, \mathfrak{b} \in\{n:$ non, $a$ : $a c c, r: r e j\}$. We define $\mathrm{A}_{\sigma}, \mathrm{v}_{0}$, and $\mathrm{f}$ as

$$
\begin{aligned}
& \mathrm{A}_{\sigma}=\left(\begin{array}{c|c}
\mathrm{U}_{\sigma, n-n} \otimes \mathrm{U}_{\sigma, n-n}^{*} & 0_{k_{1}^{2} \times k_{2}} \\
\hline \mathrm{U}_{\sigma, n-a}(1) \otimes \mathrm{U}_{\sigma, n-a}^{*}(1) & \\
\vdots & \mathrm{I}_{k_{2} \times k_{2}} \\
\mathrm{U}_{\sigma, n-a}\left(k_{2}\right) \otimes \mathrm{U}_{\sigma, n-a}^{*}\left(k_{2}\right) &
\end{array}\right), \\
& \mathrm{v}_{0}=(1,0, \ldots, 0)^{\top}, \\
& \mathrm{f}=\left(0, \ldots, 0,1_{1 \times k_{2}}\right),
\end{aligned}
$$

where

- $\mathrm{A}_{\sigma}$ is a $\left(k_{1}^{2}+k_{2}\right) \times\left(k_{1}^{2}+k_{2}\right)$ square matrix;

- $\mathrm{v}_{0}$ and $\mathrm{f}$ are $k_{1}^{2}+k_{2}$ dimensional column and row vectors, respectively;

- $\mathrm{U}_{\sigma, n-n}^{*}$ denotes the conjugate of $\mathrm{U}_{\sigma, n-n}$;

$-0_{k_{1}^{2} \times k_{2}}$ represents the $k_{1}^{2} \times k_{2}$ zero matrix;

$-\mathrm{I}_{k_{2} \times k_{2}}$ is the $k_{2} \times k_{2}$ identity matrix;

- $\mathrm{U}_{\sigma, n-a}(i)$ denotes the $i^{t h}$ row of $\mathrm{U}_{\sigma, n-a}$, and $\mathrm{U}_{\sigma, n-a}^{*}(i)$ denotes its conjugate, $\left(1 \leq i \leq k_{2}\right)$, and;

$-1_{1 \times k_{2}}$ is a $1 \times k_{2}$ row vector whose entries are all 1 's.

For a given input string $w \in \Sigma^{*}, \mathbf{w}=\$ w \$$, we will prove by induction that

$$
\mathbf{v}_{j}=(\underbrace{\left(\left|\mathbf{u}_{j}^{n}\right\rangle_{k_{1}} \otimes\left|\mathbf{u}_{j}^{n}\right\rangle_{k_{1}}^{*}\right)^{\top}}_{k_{1}^{2} \text { entries }} \mid \underbrace{P_{\mathcal{M}, \text { acc }}^{q_{k_{1}+1}}(j), \cdots, P_{\mathcal{M}, a c c}^{q_{k_{1}+k_{2}}}(j)}_{k_{2} \text { entries }})^{\top},
$$

where

$-1 \leq j \leq|\mathbf{w}|$,

$-\mathrm{v}_{j}=\left(\mathrm{A}_{\mathbf{w}_{j}}\right) \mathrm{v}_{j-1}$

- $\left|\mathbf{u}_{j}^{n}\right\rangle_{k_{1}}$ is a $k_{1}$-dimensional vector formed of the top $k_{1}$ entries of the non-halting states vector $\left|\mathbf{u}_{j}^{n}\right\rangle$ of $\mathcal{M}$ after the $j^{\text {th }}$ step and $\left|\mathrm{u}_{j}^{n}\right\rangle_{k_{1}}^{*}$ is its conjugate,

- $P_{\mathcal{M}, a c c}^{q_{k_{1}+l}}(j)$ is the probability that $\mathcal{M}$ halts by reaching the accepting state $q_{k_{1}+l}$, in the first $j$ steps of the computation $\left(1 \leq l \leq k_{2}\right)$.

$\mathrm{v}_{0}$ can be written as $\left(\left(\left|\mathbf{u}_{0}^{n}\right\rangle_{k_{1}} \otimes\left|\mathbf{u}_{0}^{n}\right\rangle_{k_{1}}^{*}\right)^{\top} \mid 0, \cdots, 0\right)^{\top}$.

For $j=1$,

$$
\mathrm{v}_{1}=\mathrm{A}_{\mathrm{w}_{1}} \mathrm{v}_{0} .
$$

The first $k_{1}^{2}$ entries of $v_{1}$ make up the vector

$$
\begin{aligned}
\left(\mathbf{U}_{\mathbf{w}_{1}, n-n} \otimes \mathbf{U}_{\mathbf{w}_{1}, n-n}^{*}\right)\left(\left|\mathbf{u}_{0}^{n}\right\rangle_{k_{1}} \otimes\left|\mathbf{u}_{0}^{n}\right\rangle_{k_{1}}^{*}\right) & =\left(\mathbf{U}_{\mathbf{w}_{1}, n-n}\left|\mathbf{u}_{0}^{n}\right\rangle_{k_{1}}\right) \otimes\left(\mathbf{U}_{\mathbf{w}_{1}, n-n}^{*}\left|\mathbf{u}_{0}^{n}\right\rangle_{k_{1}}^{*}\right) \\
& =\left(\left|\mathbf{u}_{1}^{n}\right\rangle_{k_{1}} \otimes\left|\mathbf{u}_{1}^{n}\right\rangle_{k_{1}}^{*}\right) .
\end{aligned}
$$

The remaining $k_{2}$ entries of $\mathrm{v}_{1}$ are, $1 \leq l \leq k_{2}$,

$$
\begin{aligned}
\mathbf{v}_{1}\left(k_{1}^{2}+l\right) & =\left(\mathbf{U}_{\mathbf{w}_{1}, n-a}(l) \otimes \mathbf{U}_{\mathbf{w}_{1}, n-a}^{*}(l)\right)\left(\left|\mathbf{u}_{0}^{n}\right\rangle_{k_{1}} \otimes\left|\mathbf{u}_{0}^{n}\right\rangle_{k_{1}}^{*}\right) \\
& =\left(\mathbf{U}_{\mathbf{w}_{1}, n-a}(l)\left|\mathbf{u}_{0}^{n}\right\rangle_{k_{1}}\right) \otimes\left(\mathrm{U}_{\mathbf{w}_{1}, n-a}^{*}(l)\left|\mathbf{u}_{0}^{n}\right\rangle_{k_{1}}^{*}\right) \\
& =\left(\left|\mathbf{u}_{1}^{a}\right\rangle_{k_{1}}(l) \otimes\left|\mathbf{u}_{1}^{a}\right\rangle_{k_{1}}^{*}(l)\right) \\
& =\left(\left|\mathbf{u}_{1}^{a}\right\rangle_{k_{1}}(l)\left|\mathbf{u}_{1}^{a}\right\rangle_{k_{1}}^{*}(l)\right) \\
& =P_{\mathcal{M}, a c c}^{q_{k_{1}+l}}(1) .
\end{aligned}
$$


For the inductive step, $j$,

$$
\mathrm{v}_{j}=\mathrm{A}_{\mathbf{w}_{j}} \mathrm{v}_{j-1} .
$$

The first $k_{1}^{2}$ entries of $\mathrm{v}_{j}$ make up the vector

$$
\begin{aligned}
\left(\mathbf{U}_{\mathbf{w}_{j}, n-n} \otimes \mathbf{U}_{\mathbf{w}_{j}, n-n}^{*}\right)\left(\left|\mathbf{u}_{j-1}^{n}\right\rangle_{k_{1}} \otimes\left|\mathbf{u}_{j-1}^{n}\right\rangle_{k_{1}}^{*}\right) & =\left(\mathbf{U}_{\mathbf{w}_{j}, n-n}\left|\mathbf{u}_{j-1}^{n}\right\rangle_{k_{1}}\right) \otimes\left(\mathbf{U}_{\mathbf{w}_{j}, n-n}^{*}\left|\mathbf{u}_{j-1}^{n}\right\rangle_{k_{1}}^{*}\right) \\
& =\left(\left|\mathbf{u}_{j}^{n}\right\rangle_{k_{1}} \otimes\left|\mathbf{u}_{j}^{n}\right\rangle_{k_{1}}^{*}\right) .
\end{aligned}
$$

The remaining $k_{2}$ entries of $\mathrm{v}_{j}$ are, $1 \leq l \leq k_{2}$,

$$
\begin{aligned}
\mathbf{v}_{j}\left(k_{1}^{2}+l\right) & =\left(\mathbf{U}_{\mathbf{w}_{j}, n-a}(l) \otimes \mathbf{U}_{\mathbf{w}_{j}, n-a}^{*}(l)\right)\left(\left|\mathbf{u}_{j-1}^{n}\right\rangle_{k_{1}} \otimes\left|\mathbf{u}_{j-1}^{n}\right\rangle_{k_{1}}^{*}\right)+P_{\mathcal{M}, a c c}^{q_{k_{1}+l}}(j-1) \\
& =\left(\mathbf{U}_{\mathbf{w}_{j}, n-a}(l)\left|\mathbf{u}_{j-1}^{n}\right\rangle_{k_{1}}\right) \otimes\left(\mathbf{U}_{\mathbf{w}_{j}, n-a}^{*}(l)\left|\mathbf{u}_{j-1}^{n}\right\rangle_{k_{1}}^{*}\right)+P_{\mathcal{M}, a c c}^{q_{k_{1}}+l}(j-1) \\
& =\left(\left|\mathbf{u}_{j}^{a}\right\rangle_{k_{1}}(l) \otimes\left|\mathbf{u}_{j}^{a}\right\rangle_{k_{1}}^{*}(l)\right)+P_{\mathcal{M} k_{1+l}}^{*}(j-1) \\
& =\left(\left|\mathbf{u}_{j}^{a}\right\rangle_{k_{1}}(l)\left|\mathbf{u}_{j}^{a}\right\rangle_{k_{1}}^{*}(l)\right)+P_{\mathcal{M}, a c c}^{q_{k_{1}+l}, a c c}(j-1) \\
& =P_{\mathcal{M}, a c c}^{q_{1}+l}(j) .
\end{aligned}
$$

So, it is easily followed that

$$
\mathbf{v}_{|\mathbf{w}|}=\left(\left(\left|\mathbf{u}_{|\mathbf{w}|}^{n}\right\rangle_{k_{1}} \otimes\left|\mathbf{u}_{|\mathbf{w}|}^{n}\right\rangle_{k_{1}}^{*}\right)^{\top} \mid P_{\mathcal{M}, a c c}^{q_{k_{1}+1}}(|\mathbf{w}|), \cdots, P_{\mathcal{M}, a c c}^{q_{k_{1}+k_{2}}}(|\mathbf{w}|)\right)^{\top},
$$

and

$$
\begin{aligned}
\mathrm{f} . \mathrm{v}_{|\mathbf{w}|} & =\sum_{l=1}^{k_{2}} P_{\mathcal{M}, a c c}^{q_{k_{1}+l}}(|\mathbf{w}|) \\
& =P_{\mathcal{M}, a c c}(|\mathbf{w}|) \\
& =f_{\mathcal{M}}(w) .
\end{aligned}
$$

Moreover, by using $f_{\mathcal{M}}(w)=\left(f_{\mathcal{M}}(w)\right)^{\top}$,

$$
\begin{aligned}
& f_{\mathcal{M}}(w)=\left(\mathbf{f} \mathrm{A}_{\mathbf{w}_{\mid \mathbf{w}} \mid} \mathrm{A}_{\mathbf{w}_{|\mathbf{w}|-1}} \cdots \mathrm{A}_{\mathbf{w}_{1}} \mathbf{v}_{0}\right)^{\mathrm{T}} \\
& =\left(v_{0}^{\top} A_{\Phi}^{\top}\right) A_{w_{1}}^{\top} \cdots A_{w_{|w|}}^{\top}\left(A_{\Phi}^{\top} f^{\top}\right) \\
& =v_{0}^{\prime} A_{w_{|1|}}^{\prime} \cdots A_{w_{|w|}}^{\prime} f^{\prime} \\
& =v_{0}^{\prime} A_{w}^{\prime} f^{\prime} \text {, }
\end{aligned}
$$

where $\mathrm{v}_{0}^{\prime}=\mathrm{v}_{0}^{\top} \mathrm{A}_{\Phi}^{\top}, \mathrm{f}^{\prime}=\mathrm{A}_{\Phi}^{\top} \mathrm{f}^{\top}$, and $\mathrm{A}_{w_{i}}^{\prime}=\mathrm{A}_{w_{i}}^{\top}, 1 \leq i \leq|w|$.

Since any complex number $c=a+b i$ can be represented by $2 \times 2$ real matrices

$$
c=\left(\begin{array}{cc}
a & b \\
-b & a
\end{array}\right)
$$

the complex entries of the transition matrices, the initial vector and the final vector can be eliminated [18] by replacing each entry with its equivalent $2 \times 2$ matrix.

$$
\left(\begin{array}{cc}
f_{\mathcal{M}}(w) & 0 \\
0 & f_{\mathcal{M}}(w)
\end{array}\right)=\mathrm{v}_{0}^{\prime \prime} \mathrm{A}_{w}^{\prime \prime} f^{\prime \prime}
$$

where $v_{0}^{\prime \prime}, A_{w}^{\prime \prime}, f^{\prime \prime}$ are obtained from $v_{0}^{\prime}, A_{w}^{\prime}, f^{\prime}$, respectively, after the complex entry elimination. Note that, for any symbol $\sigma \in \Sigma, \mathrm{A}_{\sigma}^{\prime \prime}$ is actually obtained from $\mathrm{A}_{\sigma}^{\prime}$ and the dimension of $\mathrm{A}_{\sigma}^{\prime \prime}$ becomes $2\left(k_{1}^{2}+k_{2}\right)$. That is, the state set, say $S$, of the machine for which $\mathrm{A}_{\sigma}^{\prime \prime}$ is a transition matrix must contain $2\left(k_{1}^{2}+k_{2}\right)$ states.

If $\bar{v}$ is the first row of $v_{0}^{\prime \prime}$ and $\bar{f}$ is the first column of $f^{\prime \prime}$, then

$$
f_{\mathcal{M}}(w)=\bar{v} A_{w}^{\prime \prime} \bar{f}
$$

Therefore, for any given input string $w \in \Sigma^{*}$,

$$
f_{\mathcal{M}}(w)=f_{\mathcal{G}}(w),
$$

where $\mathcal{G}=\left(S, \Sigma, \overline{\mathrm{v}},\left\{\mathrm{A}_{\sigma}^{\prime \prime} \mid \sigma \in \Sigma\right\}, \overline{\mathrm{f}}\right)$ is a GPFA with $O\left(n^{2}\right)$ states. 
Theorem 3. UMM equals the class of stochastic languages.

Proof. Follows from Lemma 1, Lemma 2, and Theorem 2.

We now show that, unlike classical probabilistic finite automata, allowing the tape head to "stay put" for some steps during its left-to-right traversal of the input increases the language recognition power of quantum finite automata in the unbounded error case.

Theorem 4. UMM is a proper subset of the class of languages recognized by 1.5QFA's with unbounded error.

Fig. 1. Specification of the transition function of $\mathcal{Q}$

\begin{tabular}{|c|c|c|c|}
\hline $\begin{array}{c}\text { Stages } \\
\end{array}$ & $\overline{U_{\mathrm{\Phi}}, U_{a}}$ & $U_{b}$ & $U_{\$}$ \\
\hline & $\left.\left|U_{\dot{\Phi}}\right| \overrightarrow{q_{0}}\right\rangle=\frac{1}{\sqrt{2}}\left|\overrightarrow{q_{1}}\right\rangle+\frac{1}{\sqrt{2}}\left|\overrightarrow{p_{1}}\right\rangle$ & & \\
\hline $\begin{array}{c}\mathrm{I} \\
\left(\mathrm{path}_{1}\right)\end{array}$ & $\begin{array}{l}U_{a}\left|\overrightarrow{q_{1}}\right\rangle=\frac{1}{\sqrt{2}}\left|\overrightarrow{q_{2}}\right\rangle+\frac{1}{2}\left|\downarrow A_{1}\right\rangle+\frac{1}{2}\left|\downarrow R_{1}\right\rangle \\
U_{a}\left|\overrightarrow{q_{2}}\right\rangle=\frac{1}{\sqrt{2}}\left|\overrightarrow{q_{2}}\right\rangle-\frac{1}{2}\left|\downarrow A_{1}\right\rangle-\frac{1}{2}\left|\downarrow R_{1}\right\rangle\end{array}$ & $\begin{array}{l}U_{b}\left|\overrightarrow{q_{1}}\right\rangle=\left|\downarrow R_{5}\right\rangle \\
U_{b}\left|\overrightarrow{q_{2}}\right\rangle=\left|\overrightarrow{q_{3}}\right\rangle \\
U_{b}\left|\overrightarrow{q_{3}}\right\rangle=\left|\downarrow R_{6}\right\rangle\end{array}$ & $\begin{array}{l}U_{\$}\left|\overrightarrow{q_{1}}\right\rangle=\left|\downarrow R_{3}\right\rangle \\
U_{\$}\left|\overrightarrow{q_{2}}\right\rangle=\left|\downarrow R_{4}\right\rangle \\
U_{\$}\left|\overrightarrow{q_{3}}\right\rangle=\left|\downarrow R_{5}\right\rangle\end{array}$ \\
\hline $\begin{array}{c}\mathrm{I} \\
\left(\text { path }_{2}\right)\end{array}$ & $\begin{array}{l}U_{a}\left|\overrightarrow{p_{1}}\right\rangle=\left|\downarrow w_{1}\right\rangle \\
U_{a}\left|\downarrow w_{1}\right\rangle=\frac{1}{\sqrt{2}}\left|\overrightarrow{p_{2}}\right\rangle+\frac{1}{2}\left|\downarrow A_{2}\right\rangle+\frac{1}{2}\left|\downarrow R_{2}\right\rangle \\
U_{a}\left|\overrightarrow{p_{2}}\right\rangle=\left|\downarrow w_{2}\right\rangle \\
U_{a}\left|\overrightarrow{p_{2}}\right\rangle=\frac{1}{\sqrt{2}}\left|\overrightarrow{p_{2}}\right\rangle-\frac{1}{2}\left|\downarrow A_{2}\right\rangle-\frac{1}{2}\left|\downarrow R_{2}\right\rangle\end{array}$ & $\begin{array}{l}U_{b}\left|\overrightarrow{p_{1}}\right\rangle=\left|\downarrow R_{7}\right\rangle \\
U_{b}\left|\overrightarrow{p_{2}}\right\rangle=\left|\overrightarrow{p_{3}}\right\rangle \\
U_{b}\left|\overrightarrow{p_{3}}\right\rangle=\left|\downarrow R_{8}\right\rangle\end{array}$ & $\begin{array}{l}U_{\Phi}|\overrightarrow{p 1}\rangle=\left|\downarrow R_{6}\right\rangle \\
U_{\$}\left|\overrightarrow{p_{2}}\right\rangle=\left|\downarrow R_{7}\right\rangle \\
U_{\$}\left|\overrightarrow{p_{3}}\right\rangle=\left|\downarrow R_{8}\right\rangle\end{array}$ \\
\hline $\begin{array}{c}\text { II } \\
\left(\text { path }_{1}\right)\end{array}$ & $\begin{array}{l}U_{a}\left|\overrightarrow{q_{3}}\right\rangle=\left|\downarrow w_{3}\right\rangle \\
U_{a}\left|\downarrow w_{3}\right\rangle=\frac{1}{\sqrt{2}}\left|\overrightarrow{q_{4}}\right\rangle+\frac{1}{2}\left|\downarrow A_{3}\right\rangle+\frac{1}{2}\left|\downarrow R_{3}\right\rangle \\
U_{a}\left|\overrightarrow{q_{4}}\right\rangle=\left|\downarrow w_{4}\right\rangle \\
U_{a}\left|\downarrow w_{4}\right\rangle=\frac{1}{\sqrt{2}}\left|\overrightarrow{q_{4}}\right\rangle-\frac{1}{2}\left|\downarrow A_{3}\right\rangle-\frac{1}{2}\left|\downarrow R_{3}\right\rangle\end{array}$ & $\mid \begin{array}{l}U_{b}\left|\overrightarrow{q_{4}}\right\rangle=\frac{1}{2}\left|\overrightarrow{q_{5}}\right\rangle+\frac{1}{2 \sqrt{2}}\left|\overrightarrow{a_{1}}\right\rangle+\frac{1}{2 \sqrt{2}}\left|\overrightarrow{r_{1}}\right\rangle+\frac{1}{2}\left|\downarrow A_{1}\right\rangle+\frac{1}{2}\left|\downarrow R_{1}\right\rangle \\
U_{b}\left|\overrightarrow{q_{5}}\right\rangle=\left|\downarrow R_{9}\right\rangle\end{array}$ & $\begin{array}{l}U_{\$}\left|\overrightarrow{q_{4}}\right\rangle=\left|\downarrow R_{9}\right\rangle \\
U_{\$}\left|\overrightarrow{q_{5}}\right\rangle=\frac{1}{2}\left|\downarrow A_{1}\right\rangle+\frac{1}{2}\left|\downarrow R_{1}\right\rangle\end{array} \mid$ \\
\hline $\begin{array}{c}\text { II } \\
\left(\text { path }_{2}\right)\end{array}$ & $\begin{array}{l}U_{a}|\vec{p} \overrightarrow{3}\rangle=\frac{1}{\sqrt{2}}\left|\overrightarrow{p_{4}}\right\rangle+\frac{1}{2}\left|\downarrow A_{4}\right\rangle+\frac{1}{2}\left|\downarrow R_{4}\right\rangle \\
U_{a}\left|\overrightarrow{p_{4}}\right\rangle=\frac{1}{\sqrt{2}}\left|\overrightarrow{p_{4}}\right\rangle-\frac{1}{2}\left|\downarrow A_{4}\right\rangle-\frac{1}{2}\left|\downarrow R_{4}\right\rangle\end{array}$ & $\begin{array}{l}U_{b}\left|\overrightarrow{p_{4}}\right\rangle=\frac{1}{2}\left|\overrightarrow{p_{5}}\right\rangle+\frac{1}{2 \sqrt{2}}\left|\overrightarrow{a_{1}}\right\rangle-\frac{1}{2 \sqrt{2}}\left|\overrightarrow{r_{1}}\right\rangle+\frac{1}{2}\left|\downarrow A_{2}\right\rangle+\frac{1}{2}\left|\downarrow R_{2}\right\rangle \\
U_{b}\left|\overrightarrow{p_{5}}\right\rangle=\left|\downarrow R_{10}\right\rangle\end{array}$ & $\begin{array}{l}U_{\$}\left|\overrightarrow{p_{4}}\right\rangle=\left|\downarrow R_{10}\right\rangle \\
U_{\$}\left|\overrightarrow{p_{5}}\right\rangle=\frac{1}{2}\left|\downarrow A_{2}\right\rangle+\frac{1}{2}\left|\downarrow R_{2}\right\rangle\end{array}$ \\
\hline $\begin{array}{c}\text { III } \\
\left(\text { path }_{1}\right)\end{array}$ & $\begin{array}{l}U_{a}\left|\overrightarrow{q_{5}}\right\rangle=\left|\downarrow w_{5}\right\rangle \\
U_{a}\left|\downarrow w_{5}\right\rangle=\frac{1}{\sqrt{2}}\left|\overrightarrow{q_{6}}\right\rangle+\frac{1}{2}\left|\downarrow A_{5}\right\rangle+\frac{1}{2}\left|\downarrow R_{5}\right\rangle \\
U_{a}\left|\overrightarrow{q_{6}}\right\rangle=\left|\downarrow w_{6}\right\rangle \\
U_{a}\left|\downarrow w_{6}\right\rangle=\frac{1}{\sqrt{2}}\left|\overrightarrow{q_{6}}\right\rangle-\frac{1}{2}\left|\downarrow A_{5}\right\rangle-\frac{1}{2}\left|\downarrow R_{5}\right\rangle\end{array}$ & $\left.\left|U_{b}\right| \overrightarrow{q_{6}}\right\rangle=\frac{1}{2}\left|\overrightarrow{q_{5}}\right\rangle+\frac{1}{2 \sqrt{2}}\left|\overrightarrow{a_{1}}\right\rangle+\frac{1}{2 \sqrt{2}}\left|\overrightarrow{r_{1}}\right\rangle-\frac{1}{2}\left|\downarrow A_{1}\right\rangle-\frac{1}{2}\left|\downarrow R_{1}\right\rangle$ & $U_{\$}|\overrightarrow{q b}\rangle=\left|\downarrow R_{11}\right\rangle$ \\
\hline $\begin{array}{c}\text { III } \\
\left(\text { path }_{2}\right)\end{array}$ & $\begin{array}{l}U_{a}\left|\vec{p} p_{5}\right\rangle=\frac{1}{\sqrt{2}}\left|\overrightarrow{p_{6}}\right\rangle+\frac{1}{2}\left|\downarrow A_{6}\right\rangle+\frac{1}{2}\left|\downarrow R_{6}\right\rangle \\
U_{a}\left|\overrightarrow{p_{6}}\right\rangle=\frac{1}{\sqrt{2}}\left|\overrightarrow{p_{6}}\right\rangle-\frac{1}{2}\left|\downarrow A_{6}\right\rangle-\frac{1}{2}\left|\downarrow R_{6}\right\rangle\end{array}$ & $\left.\left|U_{b}\right| \overrightarrow{p_{6}}\right\rangle=\frac{1}{2}\left|\overrightarrow{p_{5}}\right\rangle+\frac{1}{2 \sqrt{2}}\left|\overrightarrow{a_{1}}\right\rangle-\frac{1}{2 \sqrt{2}}\left|\overrightarrow{r_{1}}\right\rangle-\frac{1}{2}\left|\downarrow A_{2}\right\rangle-\frac{1}{2}\left|\downarrow R_{2}\right\rangle$ & $U_{\$}\left|\overrightarrow{p_{6}}\right\rangle=\left|\downarrow R_{12}\right\rangle$ \\
\hline $\begin{array}{c}\text { III } \\
\text { (path } \\
\text { (paccept) }\end{array}$ & $\begin{array}{l}U_{a}\left|\overrightarrow{a_{1}}\right\rangle=\frac{1}{\sqrt{2}}\left|\overrightarrow{a_{2}}\right\rangle+\frac{1}{2}\left|\downarrow A_{7}\right\rangle+\frac{1}{2}\left|\downarrow R_{7}\right\rangle \\
U_{a}\left|\overrightarrow{a_{2}}\right\rangle=\frac{1}{\sqrt{2}}\left|\overrightarrow{a_{2}}\right\rangle-\frac{1}{2}\left|\downarrow A_{7}\right\rangle-\frac{1}{2}\left|\downarrow R_{7}\right\rangle \\
U_{a}\left|\overrightarrow{a_{3}}\right\rangle=\frac{1}{\sqrt{2}}\left|\overrightarrow{a_{4}}\right\rangle+\frac{1}{2}\left|\downarrow A_{8}\right\rangle+\frac{1}{2}\left|\downarrow R_{8}\right\rangle \\
U_{a}\left|\overrightarrow{a_{4}}\right\rangle=\frac{1}{\sqrt{2}}\left|\overrightarrow{a_{4}}\right\rangle-\frac{1}{2}\left|\downarrow A_{8}\right\rangle-\frac{1}{2}\left|\downarrow R_{8}\right\rangle\end{array}$ & $\begin{array}{l}U_{b}\left|\overrightarrow{a_{2}}\right\rangle=\frac{1}{\sqrt{2}}\left|\overrightarrow{a_{3}}\right\rangle+\frac{1}{2}\left|\downarrow A_{3}\right\rangle+\frac{1}{2}\left|\downarrow R_{3}\right\rangle \\
U_{b}\left|\overrightarrow{a_{1}}\right\rangle=\left|\downarrow R_{11}\right\rangle \\
U_{b}\left|\overrightarrow{a_{4}}\right\rangle=\frac{1}{\sqrt{2}}\left|\overrightarrow{a_{3}}\right\rangle-\frac{1}{2}\left|\downarrow A_{3}\right\rangle-\frac{1}{2}\left|\downarrow R_{3}\right\rangle \\
U_{b}\left|\overrightarrow{a_{3}}\right\rangle=\left|\downarrow R_{12}\right\rangle\end{array}$ & $\begin{array}{l}U_{\$}\left|\overrightarrow{a_{1}}\right\rangle=\left|\downarrow A_{3}\right\rangle \\
U_{\$}|\vec{a}\rangle=\left|\downarrow A_{4}\right\rangle \\
U_{\$}\left|\overrightarrow{a_{2}}\right\rangle=\left|\downarrow R_{13}\right\rangle \\
U_{\$}\left|\overrightarrow{a_{4}}\right\rangle=\left|\downarrow R_{14}\right\rangle\end{array}$ \\
\hline $\begin{array}{c}\text { III } \\
\left(\text { path }_{\text {reject }}\right)\end{array}$ & $\begin{array}{l}U_{a}\left|\overrightarrow{r_{1}}\right\rangle=\frac{1}{\sqrt{2}}\left|\overrightarrow{r_{2}}\right\rangle+\frac{1}{2}\left|\downarrow A_{9}\right\rangle+\frac{1}{2}\left|\downarrow R_{9}\right\rangle \\
U_{a}\left|\overrightarrow{r_{2}}\right\rangle=\frac{1}{\sqrt{2}}\left|\overrightarrow{r_{2}}\right\rangle-\frac{1}{2}\left|\downarrow A_{9}\right\rangle-\frac{1}{2}\left|\downarrow R_{9}\right\rangle \\
U_{a}\left|\overrightarrow{r_{3}}\right\rangle=\frac{1}{\sqrt{2}}\left|\overrightarrow{r_{4}}\right\rangle+\frac{1}{2}\left|\downarrow A_{10}\right\rangle+\frac{1}{2}\left|\downarrow R_{10}\right\rangle \\
U_{a}\left|\overrightarrow{r_{4}}\right\rangle=\frac{1}{\sqrt{2}}\left|\overrightarrow{r_{4}}\right\rangle-\frac{1}{2}\left|\downarrow A_{10}\right\rangle-\frac{1}{2}\left|\downarrow R_{10}\right\rangle\end{array}$ & $\begin{array}{l}U_{b}\left|\overrightarrow{r_{2}}\right\rangle=\frac{1}{\sqrt{2}}\left|\overrightarrow{r_{3}}\right\rangle+\frac{1}{2}\left|\downarrow A_{4}\right\rangle+\frac{1}{2}\left|\downarrow R_{4}\right\rangle \\
U_{b}\left|\overrightarrow{r_{1}}\right\rangle=\left|\downarrow R_{13}\right\rangle \\
U_{b}\left|\overrightarrow{r_{4}}\right\rangle=\frac{1}{\sqrt{2}}\left|\overrightarrow{r_{3}}\right\rangle-\frac{1}{2}\left|\downarrow A_{4}\right\rangle-\frac{1}{2}\left|\downarrow R_{4}\right\rangle \\
U_{b}\left|\overrightarrow{r_{3}}\right\rangle=\left|\downarrow R_{14}\right\rangle\end{array}$ & $\begin{array}{l}U_{\$}|\vec{r} \vec{r}\rangle=\left|\downarrow R_{15}\right\rangle \\
U_{\$}|\vec{r}\rangle=\left|\downarrow R_{16}\right\rangle \\
U_{\$}\left|\overrightarrow{r_{2}}\right\rangle=\left|\downarrow R_{17}\right\rangle \\
U_{\$}|\vec{r}\rangle=\left|\downarrow R_{18}\right\rangle\end{array}$ \\
\hline
\end{tabular}

Proof. By the definition of 1.5QFA's and Theorem 3, every stochastic language can be recognized with unbounded error by 1.5QFA's. We will show that the context-free nonstochastic language [19]

$$
\mathcal{L}_{1}=\left\{a^{x} b a^{y_{1}} b a^{y_{2}} b \cdots a^{y_{t}} b \in\{a, b\}^{*} \mid x, t, y_{1}, \cdots, y_{t} \in \mathbb{Z}^{+} \text {and }, \exists k(1 \leq k \leq t), x=\sum_{i=1}^{k} y_{i}\right\}
$$

can be recognized with unbounded error by a $1.5 \mathrm{QFA}$ with cutpoint $\frac{1}{2}$. Consider the $1.5 \mathrm{QFA} \mathcal{Q}=$ $\left(Q, \Sigma, \delta, Q_{a c c}, Q_{r e j}\right)$, where $\Sigma=\{a, b\}$ and the state sets are as follows: $Q_{\text {non }}=\left\{\overrightarrow{q_{0}}\right\} \cup\left\{\overrightarrow{q_{i}} \mid 1 \leq i \leq 6\right\} \cup\left\{\overrightarrow{p_{i}} \mid 1 \leq i \leq 6\right\} \cup\left\{\overrightarrow{a_{i}} \mid 1 \leq i \leq 4\right\} \cup\left\{\overrightarrow{r_{i}} \mid 1 \leq i \leq 4\right\}$

$\cup\left\{\downarrow w_{i} \mid 1 \leq i \leq 6\right\}, Q_{a c c}=\left\{\downarrow A_{i} \mid 1 \leq i \leq 10\right\}$, and $Q_{r e j}=\left\{\downarrow R_{i} \mid 1 \leq i \leq 18\right\}$.

Let each $U_{\sigma}$ act as indicated in Figure 1, and extend each to be unitary. Let $\delta$ be related to the $U_{\sigma}$ as described in Section 2. 
Machine $\mathcal{Q}$ starts computation on symbol $\oplus$ by branching into two paths, path $_{1}$ and path $_{2}$, with equal probability. Each path and their subpaths, to be described later, check whether the input is of the form $\left(a a^{*} b\right)\left(a a^{*} b\right)\left(a a^{*} b\right)^{*}$. The different stages of the program indicated in Figure 1 correspond to the subtasks of this regular expression check. Stage I ends successfully if the input begins with $\left(a a^{*} b\right)$. Stage II checks the second $\left(a a^{*} b\right)$. Finally, Stage III controls whether the input ends with $\left(a a^{*} b\right)^{*}$.

The reader will note that many transitions in the machine are of the form

$$
U_{\sigma}\left|q_{i}\right\rangle=|\psi\rangle+\alpha\left|A_{k}\right\rangle+\alpha\left|R_{k}\right\rangle
$$

where $|\psi\rangle$ is a superposition of configurations such that $\langle\psi \mid \psi\rangle=1-2 \alpha^{2}, A_{k} \in Q_{a c c}, R_{k} \in Q_{r e j}$. The equal-probability transitions to the "twin halting states" $A_{k}$ and $R_{k}$ are included to ensure that the matrices are unitary, without upsetting the "accept/reject balance" until a final decision about the membership of the input in $\mathcal{L}_{1}$ is reached. If the regular expression check mentioned above fails, each path in question terminates in a rejecting configuration, and the overall probability of acceptance of the machine turns out to be less than $\frac{1}{2}$. If the input is indeed of the form $\left(a a^{*} b\right)\left(a a^{*} b\right)\left(a a^{*} b\right)^{*}$, the acceptance probability is at least $\frac{1}{2}$, and whether $\frac{1}{2}$ will be exceeded or not depends on the following additional tasks performed by the computation paths in order to test for the equality mentioned in the definition of $\mathcal{L}_{1}$ :

1. path $_{1}$ walks over the $a$ 's at the speed of one tape square per step until reading the first $b$. After that point, path $_{1}$ pauses for one step over each $a$ before moving on to the next symbol.

2. path $_{2}$ pauses for one step over each $a$ until reading the first $b$. After that point, path 2 walks over each $a$ at the speed of one square per step.

3. On each $b$ except the first one, path 1 and path $_{2}$ split to take the following two courses of action with equal probability:

(a) In the first alternative, path $_{1}$ and path $_{2}$ perform a two-way quantum Fourier transform (QFT) [13]:

i. The targets of the QFT are two new computational paths, i.e., path accept $_{\text {and }}$ path reject $_{\text {. }}$. Disregarding the equal-probability transitions to the twin halting states mentioned above, the QFT is realized as:

$$
\begin{aligned}
& \text { path }_{1} \rightarrow \frac{1}{\sqrt{2}} \text { path }_{\text {accept }}+\frac{1}{\sqrt{2}} \text { path }_{\text {reject }} \\
& \text { path }_{2} \rightarrow \frac{1}{\sqrt{2}} \text { path }_{\text {accept }}-\frac{1}{\sqrt{2}} \text { path }_{\text {reject }}
\end{aligned}
$$

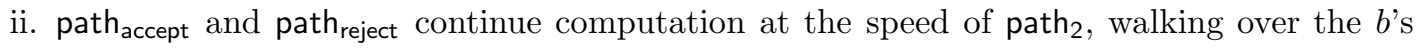
without performing the QFT any more.

(b) In the second alternative, path $_{1}$ and path $_{2}$ continue computation without performing the QFT.

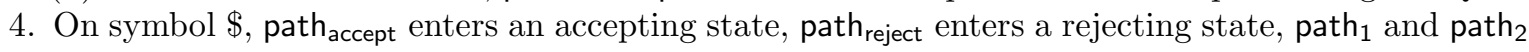
enter accepting and rejecting states with equal probability.

Suppose that the input is of the form

$$
w=a^{x} b a^{y_{1}} b a^{y_{2}} b \cdots a^{y_{t}} b
$$

where $x, t, y_{1}, \cdots, y_{t} \in \mathbb{Z}^{+}$.

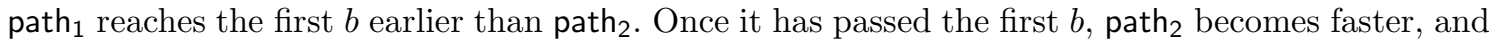

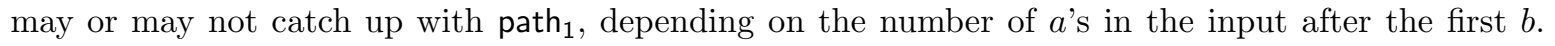
The two paths can meet on the symbol following the $x^{\prime}$ th $a$ after the first $b$, since at that point path

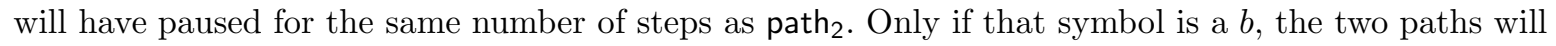
perform a QFT in the same place and at the same time. To paraphrase, if there exists a $k(1 \leq k \leq t)$ such that $x=\sum_{i=1}^{k} y_{i}$, path and path $_{2}$ meet over the $(k+1)^{t h} b$ and perform the QFT at the same step. If there is no such $k$, the paths either never meet, or meet over an $a$ without a QFT. 
The path $\mathrm{accept}_{\text {and }}$ path $\mathrm{reject}_{\mathrm{s}} \mathrm{s}$ that are offshoots of path ${ }_{1}$ continue their traversal of the string faster

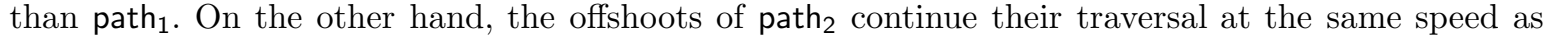
path $_{2}$.

By definition, the twin halting states reached during the computation contribute equal amounts to the acceptance and rejection probabilities. path $_{1}$ and path $_{2}$ accept and reject equiprobably when they reach the end of the string. If path ${ }_{1}$ and path ${ }_{2}$ never perform the QFT at the same time and in the same position, every QFT produces two equal-probability paths which perform identical tasks, except that one accepts and the other one rejects at the end.

The overall acceptance and rejection probabilities are equal, $\frac{1}{2}$, unless a path reject $_{\text {with positive }}$ amplitude and a path $_{\text {reject }}$ with negative amplitude can meet and therefore cancel each other. In such a case, the surviving path accept 's will contribute the additional acceptance probability that will tip the balance. As described above, such a cancellation is only possible when path ${ }_{1}$ and path $_{2}$ perform the QFT together.

Therefore, if $w \in \mathcal{L}_{1}$, the overall acceptance probability is greater than $\frac{1}{2}$. If $w \notin \mathcal{L}_{1}$, the overall acceptance probability does not exceed $\frac{1}{2}$.

\section{Concluding Remarks}

In this paper, we examined the capabilities of measure-many (Kondacs-Watrous) quantum finite automata in the unbounded error setting. We gave a full characterization of the class of languages recognized by one-way QFA's of this kind; they turn out to recognize all and only the stochastic languages. We also showed that allowing the tape head to "stay put" for some steps during its left-to-right traversal of the input increases the language recognition power of quantum finite automata in the unbounded error case. This means that two-way (and even "1.5-way") QFA's are strictly more powerful than 1QFA's; whereas 2PFA's are known to be equivalent in power to 1PFA's [12] in this setting. Since two-way finite automata with suitable restrictions (which do not affect our results) on the transition amplitudes are equivalent to Turing machines restricted to constant space bounds, this answers a question of Watrous [25] about the relationship between the computational powers of probabilistic Turing machines and quantum Turing machines with space bounds less than $\log n$; QTM's are strictly more powerful than PTM's in this case.

Theorem 3 has some obvious ramifications about upper bounds on the numbers of states of KWQFA's that recognize regular languages. All the well-known results $[17,22]$ about the "state economy" provided by NFA's and PFA's over DFA's carry over easily to KWQFA's. Lemma 1 can be specialized to show that an $n$-state DFA can be simulated by an unbounded-error KWQFA with just $O(n)$ states, and cut-point 0 .

Other types of QFA's $[2,5,9,11,15,18,20,21]$ have been defined in the literature. The class of languages recognized with unbounded error by measure-once QFA's $[7,18]$ has been shown to be a proper subset of the stochastic languages. The relationship of UMM to the classes of languages recognized by the other QFA variants with unbounded error is an open question. Another question left open in this work is the relationship between the computational powers of 1.5QFA's and 2QFA's.

\section{Acknowledgement}

We thank Rūsinsš Freivalds for his helpful answers to our questions regarding nonstochastic languages.

\section{References}

1. Masami Amano and Kazuo Iwama. Undecidability on quantum finite automata. In STOC '99: Proceedings of the thirty-first annual ACM symposium on Theory of computing, pages 368-375, New York, NY, USA, 1999. ACM. 
2. Andris Ambainis, Martin Beaudry, Marats Golovkins, Arnolds Ķikusts, Mark Mercer, and Denis Thérien. Algebraic results on quantum automata. Theory of Computing Systems, 39(1):165-188, 2006.

3. Andris Ambainis and Rūsinsš Freivalds. 1-way quantum finite automata: strengths, weaknesses and generalizations. In FOCS'98: Proceedings of the 39th Annual Symposium on Foundations of Computer Science, pages 332-341, Palo Alto, California, 1998.

4. Andris Ambainis and John Watrous. Two-way finite automata with quantum and classical states. Theoretical Computer Science, 287(1):299-311, 2002.

5. Aleksandrs Belovs, Ansis Rosmanis, and Juris Smotrovs. Multi-letter reversible and quantum finite automata. In Tero Harju, Juhani Karhumäki, and Arto Lepistö, editors, Developments in Language Theory, volume 4588 of $L N C S$, pages 60-71. Springer Berlin / Heidelberg, 2007.

6. Ethan Bernstein and Umesh Vazirani. Quantum complexity theory. SIAM Journal on Computing, 26(5), 1997.

7. Alberto Bertoni and Marco Carpentieri. Analogies and differences between quantum and stochastic automata. Theoretical Computer Science, 262(1-2):69-81, 2001.

8. Alberto Bertoni and Marco Carpentieri. Regular languages accepted by quantum automata. Information and Computation, 165(2):174-182, 2001.

9. Alberto Bertoni, Carlo Mereghetti, and Beatrice Palano. Quantum computing: 1-way quantum automata. In Zoltán Ésik and Zoltán Fülöp, editors, Developments in Language Theory, volume 2710 of LNCS, pages 1-20. Springer Berlin / Heidelberg, 2003.

10. Alex Brodsky and Nicholas Pippenger. Characterizations of 1-way quantum finite automata. SIAM Journal on Computing, 31(5):1456-1478, 2002.

11. Massimo Pica Ciamarra. Quantum reversibility and a new model of quantum automaton. In $F C T$ '01: Proceedings of the 13th International Symposium on Fundamentals of Computation Theory, pages 376-379, London, UK, 2001. Springer-Verlag.

12. Jānis Kaņeps. Stochasticity of the languages acceptable by two-way finite probabilistic automata. Diskretnaya Matematika, 1:63-67, 1989. (Russian).

13. Attila Kondacs and John Watrous. On the power of quantum finite state automata. In FOCS'97: Proceedings of the 38th Annual Symposium on Foundations of Computer Science, pages 66-75, Miami, Florida, 1997.

14. Yu. I. Kuklin. Two-way probabilistic automata. Avtomatika i vyčistitelnaja tekhnika, 5:36-36, 1973. (Russian).

15. Mark Mercer. Applications of Algebraic Automata Theory to Quantum Finite Automata. PhD thesis, McGill University, 2007.

16. Mark Mercer. Lower bounds for generalized quantum finite automata. The 2nd International Conference on Language and Automata Theory and Applications, 2008.

17. A. R. Meyer and M. J. Fischer. Economy of description by automata, grammars, and formal systems. pages 188-191, East Lansing, MI, USA, 1971. 12th Annual Symposium on Switching and Automata Theory.

18. Cristopher Moore and James P. Crutchfield. Quantum automata and quantum grammars. Theoretical Computer Science, 237(1-2):275-306, 2000.

19. Masakazu Nasu and Namio Honda. A context-free language which is not acceptable by a probabilistic automaton. Information and Control, 18(3):233-236, 1971.

20. Ashwin Nayak. Optimal lower bounds for quantum automata and random access codes. In FOCS '99: Proceedings of the 40th Annual Symposium on Foundations of Computer Science, pages 369-376, Washington, DC, USA, 1999. IEEE Computer Society.

21. Kathrin Paschen. Quantum finite automata using ancilla qubits. Technical report, University of Karlsruhe, 2000.

22. Azaria Paz. Introduction to Probabilistic Automata. Academic Press, New York, 1971.

23. Michael O. Rabin. Probabilistic automata. In Edward F. Moore, editor, Sequential Machines, chapter 4, pages 98-114. Addison-Wesley Publishing Company, Inc., 1964.

24. Paavo Turakainen. Generalized automata and stochastic languages. Proceedings of the American Mathematical Society, 21:303-309, 1969.

25. John Watrous. Space-bounded quantum complexity. Journal of Computer and System Sciences, 59(2):281326, 1999. 